\title{
Fast Analysis of Scalable Video for Adaptive Browsing Interfaces
}

\author{
Marta Mrak, Janko Ćalić, Ahmet Kondoz \\ The University of Surrey, Guildford GU2 7XH, UK
}

\begin{abstract}
Driven by a high demand for user-centred video interfaces and recent advances in scalable video coding technology, this work introduces a novel framework for video browsing by utilising inherently hierarchical compressed-domain features of scalable video and efficient dynamic video summarisation. This approach enables instant adaptability of generated video summaries to user requirements, available channel bandwidth as well as display size. By utilising compressed domain features an efficient hierarchical analysis of motion activity at different layers of complexity is achieved. Exploiting a contour evolution algorithm, a scale space of temporal video descriptors is generated, enabling dynamic video summarisation in real-time. Given the spatial resources of terminal display and generated video summary, the final browsing layout is generated utilising an unsupervised robust spectral clustering technique and a fast discrete optimisation algorithm. Results show excellent scalability of the video browsing interface and good algorithm efficiency.
\end{abstract}

Key words: scalable video coding, compressed domain analysis, video summarisation, HCI

PACS: 43.60.Dh, 29.50.+v

\section{Introduction}

Having experienced a widespread proliferation of video streaming applications, such as YouTube and Google video, and associated web-based services and communities, the importance of user centred interfaces for video browsing has increased remarkably. In addition, recent advances in video coding technology

Email addresses: m.mrak@surrey.ac.uk (Marta Mrak), j.calic@surrey.ac.uk (Janko Ćalić), a.kondoz@surrey.ac.uk (Ahmet Kondoz). 
targeting inherent adaptability to different networking and application contexts, i.e. scalable video coding technology, brought a plethora of possibilities to deliver video information in a user centred manner.

Scalable coding technology is highly suitable for applications where compressed video parameters can be selected dynamically, i.e. without using expensive and time consuming transcoding. At the same time it shows high efficiency in terms of compression ratio and quality [1], [2], [3]. Moreover, with low-complexity adaptation capability, scalable video coding provides adjustment to user preferences, different devices and heterogeneous networks as well as iterative delivery platforms by means of multiple-point adaptation capabilities.

Besides advanced methods that enable scalability features, scalable video encoding is based on algorithms that provide high compression. To achieve compression the advanced temporal and spatial decorrelation techniques have to be applied. As in conventional non-scalable approaches, temporal decorrelation of video frames is performed using temporal decomposition driven by motion information. Particularly that motion information is the one that captures the main temporal properties of videos and it can be used for video analysis. It is well known that video descriptors computed from compressed domain are highly correlated to the subjective ground truth [4] [5] [6] [7]. In this paper we use this fact to build a video content analysis framework which is scalable in two ways - analysis of scalable content is targeted and the analysis itself is scalable depending on user request and available content parameters.

Utilisation of motion information is a central feature used for event driven temporal analysis of videos. Initial work has been exploiting motion features extracted from the dense optical flow [8]. Targeting fast and efficient processing, compressed domain motion features have been extensively utilised in video analysis, especially in video summarisation and indexing [9]. On the other hand, using standardised motion activity features, such as MPEG-7 motion activity descriptor, methods given in [7] and [10] target integration of extracted features in larger indexing and retrieval frameworks. In addition, excitement modelling has been introduced to improve quality of summarisation using overall motion activity in a given frame sequence [11]. Finally, more complex features expressed trough motion activity, such as visual attention modelling, have been employed in user centred video skimming [12].

There have been numerous approaches to analysis of temporal video features. Treating frames as points in the multi-dimensional feature space, clustering approaches extract keyframes as centres of clusters generated by applying various grouping algorithms [13]. In order to improve the robustness of the clustering, feature space pre-processing via principal component analysis can be applied [14]. Similarly, by using the multi-dimensional feature space rep- 
resentation, curve simplification approach generates a set of points used to determine the most salient key-frames. This approach optimises the associated removal cost of of the remaining points by using curve simplification algorithms such as the binary curve splitting [15] or discrete contour evolution [16] [17]. Finally, selection of the most representative set of key-frames can be achieved by following the sequence reconstruction error approach [18], that optimises the capability of the generated set to reconstruct the original video sequence.

In order to generate user-centred video summaries and thus enable intuitive browsing, various ways of video representation and summarisation have been proposed. In [19] an unsupervised clustering method based on HSV colour features has been proposed, where the frame closest to the cluster centre is chosen as the key frame representative for a given video shot. Utilising cluster-validity analysis to remove the visual content redundancy among video frames using an unsupervised procedure is proposed in [20]. Recently, in [13] a normalised cut algorithm has been emplyed to globally and optimally partition the graph representation into video clusters and describe the evolution and perceptual importance of a video segment.

This work makes a shift towards more user-centred summarisation and browsing of large video collections by exploiting the narrative structure of comics to generate easily-readable visual summaries. Comics are the most prevalent medium that expresses notion of time through a sequence of spatially structured images. The proposed system links the temporal flow of video sequence with the spatial position of key-frames laid out as panels in a comic strip [21]. This approach differentiates our work from more typical reversestoryboarding [22] or video summarisation approaches. It achieves better spatial compactness while maintaining the notion of temporal structure.

There have been attempts to utilise the form of comics as a medium for visual summarisation of videos. In [23] a layout algorithm that optimises the ratio of white space left and approximation error of the frame importance function is proposed. Following a similar approach, the work presented in [24] introduces a number of heuristic rules to optimise the layout algorithm. However, due to an inherently difficult optimisation, these attempts failed to develop a feasible layout algorithm. In order to uncover the underpinning structure of the key-frame data in an unsupervised manner, our work exploits K-way spectral clustering method [25] in perceptual grouping of extracted key-frames using locally scaled affinity matrix [26].

The work presented in this paper introduces a number of novel approaches for video analysis and summarisation. Targeting efficient browsing of large video databases, a fast and adaptable algorithm for representation of videos in a form of a comic-like summary has been developed. Proposed video anal- 
ysis algorithm is of a low complexity since it uses compressed domain data hierarchically organised motion information from compressed videos. Avoiding full video decoding, the analysis algorithm decompresses only the motion information from targeted temporal decomposition level. Proposed solution handles motion models with variable size blocks and analyses inter coded motion units, both unidirectionally and bidirectionally predicted. Therefore it is suitable for analysis for videos encoded in different formats and with different compression settings. Suitable measure of motion activity is defined and used as a base for further video summarisation. Using a fast and robust geometrical curve simplification algorithm, a scale space of temporal descriptors based on the motion activity metric is generated. Using either automatic or user driven algorithm sensitivity, a set of most representative key-frames is created as a visual summary of the analysed video. In order to generate an intuitive and yet compact video browsing interface, our approach introduces a novel solution based on dynamic programming (DP) and shows that the adopted sub-optimal approach achieves nearly optimal layout results in real-time. In addition, the presented algorithm applies a new approach to the estimation of key-frame sizes in the final layout by exploiting a spectral clustering methodology coupled with a specific cost function that balances between good content representability and discovery of unanticipated content. Furthermore, a robust unsupervised estimation of number of clusters is introduced. The evaluation results compared to existing methods of video summarisation [24] [23] showed substantial improvements in terms of algorithm efficiency, quality of optimisation, and possibility of swiftly generating much larger summaries.

In order to facilitate adaptable and hierarchical analysis in an efficient manner, utilisation of scalable video coding technology is presented in Section 2. An algorithm for temporal video analysis used in order to extract the most representative hierarchical video summary is described in Section 3. Section 4 introduces a novel browsing interface that optimises generated visual representation to available spatial resources. The results of the algorithms presented are presented in Section 5, followed by some final conclusions.

\section{Scalable Video Coding}

The main feature of scalable video coding is low-cost adaptation of the compressed bit-stream. Avoiding costly and computationally complex transcoding, which is typical for conventional video coding systems, the scalable video coding has been recognised as a straightforward solution for a broad range of applications including video streaming, surveillance systems, content management systems, broadcasting. Development of this already mature technology originates from wavelet-based image [27] [28] and video coding [29] [30] [31] [32]. Most recent improvements of methods that support scalability have paved a 
way to standardisation of block-based scalable video coding within state-ofthe-art MPEG-4 AVC/H.264 video coding standard [33] [34].

As in traditional video compression approaches, scalable video coding frameworks use motion compensation. Corresponding motion vectors are suitable for characterisation of general visual dynamics, since they convey the overall user impression of motion activity and perception of main events in the sequence. Moreover, different scales of motion information, as embedded in scalable video stream, can provide advanced options for video analysis.

\subsection{Temporal decomposition}

In addition to the scalable video codec ability to produce layered or fully embedded bit-stream enabling efficient adaptation, the most critical part of a video codec is the ability to exploit temporal redundancies. Since neighbouring frames in a video sequence are similar, a frame can be represented with data from already encoded neighbouring frames. In this process, which is called motion compensation, a significant part of the temporal redundancy can be removed. In scalable video coding the order in which motion compensation is performed on video frames is often called temporal decomposition. Temporal decomposition has to be flexible enough to support adaptation to different decoding points. Different decoding points are related to types of scalability that are targeted. Those are usually the basic types of scalability spatial (adaptation to lower frame resolution), quality (adaptation to lower bit-rates) and temporal (reducing number of frames) scalabilities, as well as their combinations. Between different scalability types, the rest of presented discussion is mostly related to the temporal scalability because of its connection to temporal decomposition and targeted analysis of its motion information.

An example showing temporal decomposition in scalable video coding is presented in Figure 1. With a target to produce a bit-stream whose selected parts provide videos of different frame rates (in Figure 1 available temporal scales of compressed video are $25 / 2^{t} \mathrm{~Hz}$, where $t=0,1, \ldots, 4$ and $25 \mathrm{~Hz}$ is the frame-rate of the original video) iterative motion compensation levels are applied to the input video. To achieve an embedded bit-stream with $T=5$ different temporal levels, $T-1$ levels of temporal decomposition are needed. In the particular example from Figure 1, four levels of temporal decomposition are performed on the original frames, enabling hierarchy of compressed domain data leading to layered bit-stream modelling.

The constraint applied in this process is that those frames that will not be available at the decoder for decoding of the lower frame-rate sequence must not be used for temporal prediction at higher decomposition level at the en- 


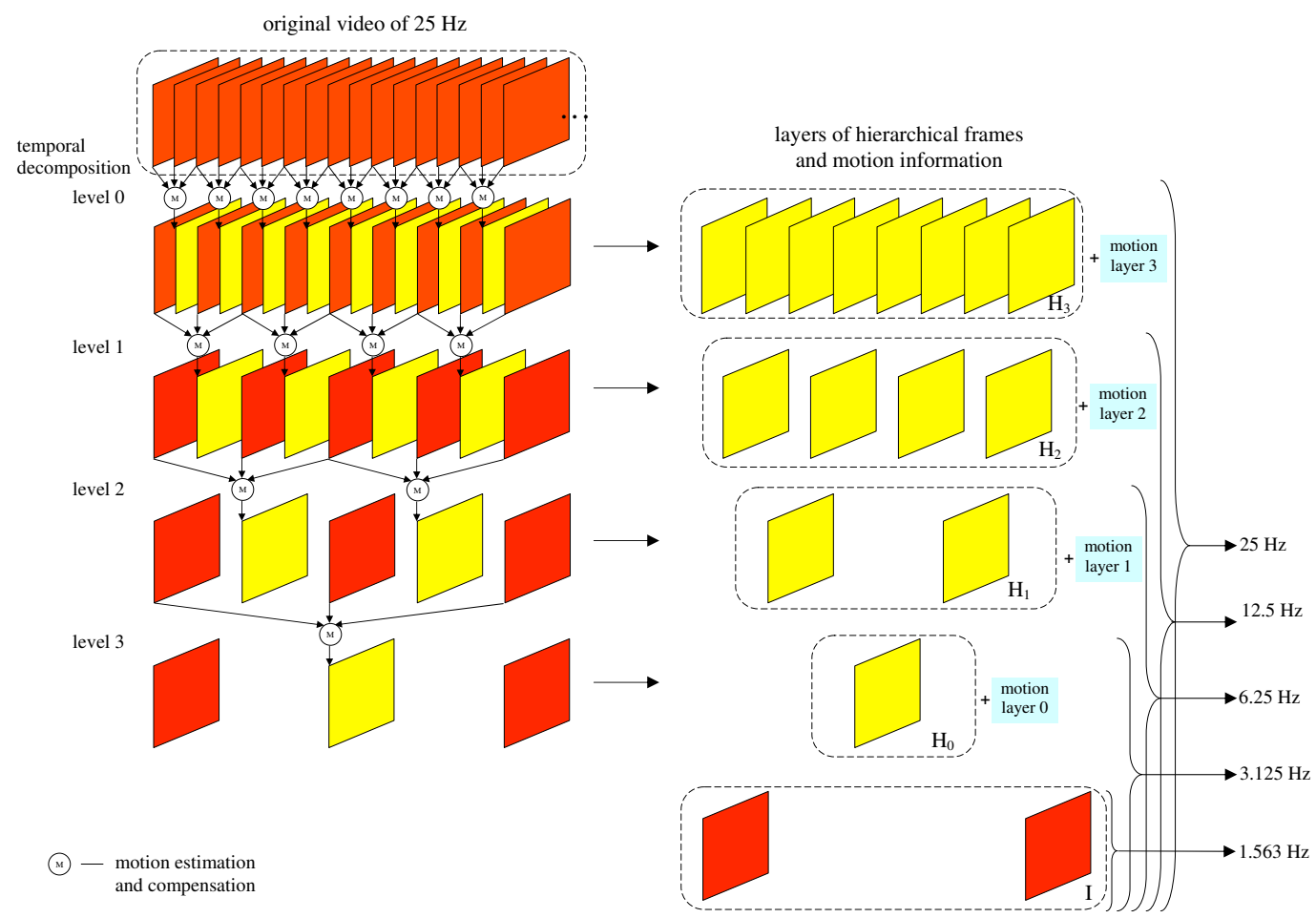

Fig. 1. Temporal decomposition and resulting hierarchical frames and motion information

coder. In the example from Figure 1 that constraint is applied by motion compensation of only every second frame at each level.

Motion compensation with constrains needed for temporal scalability is in scalable video coding often regarded as motion aligned filtering or motioncompensated temporal filtering (MCTF) [1]. Although filtering suggests that low-pass filtering has to be applied, it can be simply achieved by temporal subsampling. Additionally, if motion compensation from both neighbouring frames is allowed, motion compensated frames in temporal decomposition correspond to bidirectionally predicted frames, as in standard video coding [35].

The results of temporal decomposition are motion information, including motion vectors, and texture information, which includes intra-coded and compensated frames. In the example from Figure 1, five different frame types have resulted from temporal decomposition - I frames (or intra-coded frames) and compensated $\mathrm{H}$ frames. Texture information usually demands a significant portion of the bit-stream while motion vectors require just a small fraction. However, the motion information carries, from the analysis point of view, most significant knowledge about the video. Moreover, in scalable video coding it is already classified into hierarchical levels that can be used for analysis. 


\subsection{Motion information in video coding}

Motion compensation in video coding provides compression by removal of similarities between frames. A frame that is being compensated is divided into motion units, each of which is associated with suitable descriptors of prediction (motion modes and motion vectors) during motion estimation process. The prediction descriptors define the mode of prediction - either the temporal prediction (inter modes) or spatial or no prediction (intra modes). Motion vectors, as descriptors of the displacements between frames, are associated to units in the inter modes. Block-based motion models, utilised in this work, are used in practical realisations because of their low complexity implementation [36] [37]. Although the most efficient motion compensation is utilised with smaller units, providing for higher precision in motion description, the balance between final motion and texture rate has to meet the target coding demands [38]. Therefore the motion estimation techniques used in video coding do not aim to find a motion representation that achieves the best prediction at the encoder side, but rather aim for higher compression. Another limitation of motion estimation in video coding is related to complexity constrains. In order to achieve real-time encoding the accuracy of the most expensive process, motion estimation, is often sacrificed. Nevertheless, motion estimation in video coding does provide good estimates that lead to high compression.

In order to define motion descriptors used in the proposed framework, motion inter modes are here described briefly. As depicted in Figure 1, in general the frames can be bidirectionally predicted. However, actual prediction is decided on a base of motion units depending on frames that would be available at the decoder at the time of frame synthesis as well as on the rate-distortion optimisation.

Bidirectional prediction mode is associated to those motion units that are compensated by a signal that is obtained by averaging signals from both previous and subsequent frames selected in direction of two motion vectors - forward and backward motion vectors. On the other hand, unidirectional prediction mode means prediction from only one direction. In that case there is only one motion vector per motion unit - either forward or backward motion vector.

Since the mode of prediction for each motion unit is optimised for compression, in general there is no much correlation between subjectively perceived activities in videos and measure obtained only from motion mode. Moreover, decision on intra coding of certain units is also decided in rate-distortion optimisation. Therefore in the motion activity measure defined in the following section the intra coded units are not included. 


\section{$3 \quad$ Video Analysis}

In the proposed framework the video analysis is performed over the activity measure which we define on a frame bases. Low-complexity computation of the activity measure is achieved using compressed domain data. The motion information available from the compressed video is obtained using only partial decoding.

\subsection{Metric extraction}

For each frame an activity metric is extracted using motion information available for given frame. Only blocks to which motion vectors are associated (inter coded block) are taken into account. For the observed frame a set $\Phi$ is defined which consists of inter predicted block $\mathrm{B}_{i}$ such that $i=0, \ldots, N_{\Phi}-1$, where $N_{\Phi}=\operatorname{card}(\Phi)$ is the number of elements in $\Phi$. In order to provide a single evaluation methodology for both unidirectional prediction modes as well as for bidirectional prediction, the following measure of motion is derived for each block:

$$
m(i)=\left\{\begin{array}{l}
\frac{1}{2} \cdot\left(\left|\overrightarrow{m v}_{\mathrm{F}}(i)\right|+\left|\overrightarrow{m v}_{\mathrm{B}}(i)\right|\right), \text { for bidirectional prediction } \\
\left|\overrightarrow{m v}_{\mathrm{F}}(i)\right|, \text { for unidirectional forward prediction } \\
\left|\overrightarrow{m v}_{\mathrm{B}}(i)\right|, \text { for unidirectional backward prediction }
\end{array}\right.
$$

where $\left|\overrightarrow{m v}_{\mathrm{F}}(i)\right|$ and $\left|\overrightarrow{m v}_{\mathrm{B}}(i)\right|$ represent amplitudes of forward (F) and backward (B) motion vectors associated to $\mathrm{B}_{i}$. For the current frame the overall activity measure, $\alpha$, corresponds to standard deviation and is defined as:

$$
\alpha=\left(\frac{1}{N_{\Phi}} \cdot \sum_{i=0}^{N_{\Phi}-1}(m(i)-\bar{m})^{2}\right)^{1 / 2}
$$

where $\bar{m}$ is an average of motion measures for inter coded blocks in a given frame. The average motion measure is computed as:

$$
\bar{m}=\frac{\sum_{i=0}^{N_{\Phi}-1}(m(i) \cdot w(i))}{N_{\Phi} \cdot \sum_{i=0}^{N_{\Phi}-1} w(i)}
$$

where weighting factor $w(i)$ corresponds to the size of $\mathbf{B}_{i}$. In this way the supported for motion models with variable block sizes is achieved. 


\subsection{Discrete Contour Evolution}

Extraction of activity metric can be performed using motion information from all bit-stream layers. However, since it is expected that at different scales the activity measures will be correlated, the analysis starts at the lowest scale in order to achieve fast extraction and processing. At the lowest scale, for each frame at the time instance $t$ a motion activity measure $\alpha(t)$ is calculated. In order to generate a scalable temporal descriptor $\gamma(t)$ that facilitates dynamic extraction of key-frames from the sequence, difference metric $\alpha(t)$ needs to be simplified in a way that spurious and small changes are discarded without any influence to the main features of the metrics curve. Discrete Curve Evolution (DCE) [16] efficiently achieves this requirement: it leads to the simplification of curve complexity with no peak rounding effects and no dislocation of relevant features. The relevance measure $K$, stable with respect to noisy deformations, is given as follows:

$$
K\left(s_{t}\right)=\left(\delta_{t} \tau_{t}+\delta_{t+1} \tau_{t+1}\right)
$$

The curve evolution process is guided by a relevance order. A relevance measure value $K\left(s_{t}\right)$ is assigned to every pair of two adjacent line segments $s_{t}$ and $s_{t+1}$ in a decomposition of a given curve $S=\bigcup s_{t}, \forall t$. It represents the significance of the contribution of arc $s_{t} \cup s_{t+1}$ to the shape of $S$. The pairs of adjacent line segments are ordered with respect to this significance cost, and the least significant arc is replaced by a new linear segment $s_{t-1} \cup s_{t+1}$. As given in Equation 4, the linearization cost $K\left(s_{t}\right)$ of any supported arc $s_{t} \cup s_{t+1}$ is directly proportional to the area below the arc, depicted in Figure 2.

At each stage of simplification, i.e. scale space, the relevance measure $K\left(s_{t}\right)$ is iteratively updated. The optimal complexity level of the temporal descriptor is calculated as:

$$
K_{\text {opt }}=\bar{K}-\log \kappa \cdot \sigma_{K}
$$

where $\bar{K}$ is the mean of $K, \sigma_{K}$ is its standard deviation, while the parameter $\kappa$ controls the sensitivity of the event detection and is driven by the application requirements.

Key-frame positions are determined by the local minima in the temporal descriptor at the scale of $K=K_{\text {opt }}$. Being located at the local troughs of motion activity, the key-frames will have maximum probability of avoiding motion blur and other artefacts due to object motion or camera work. In addition, the most representative information will be conveyed by the key-frames in areas with no camera work, since the cameraman tends to focus on the main object 


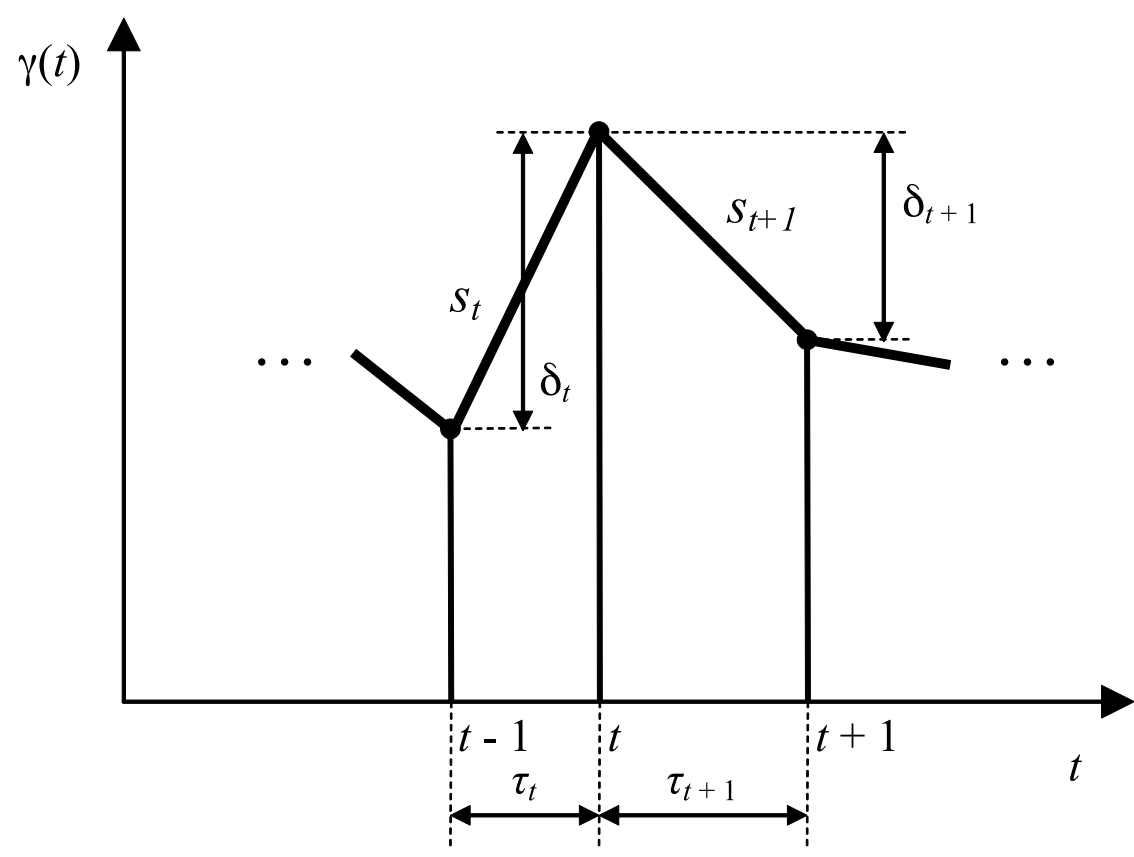

Fig. 2. Variables used in the calculation of cost function $K\left(s_{t}, s_{t+1}\right)$

of interest using a static camera. In case the level of detail required cannot be achieved by using DCE simplification, i.e. initial cost of DCE simplification is too high, algorithm switches to a higher complexity level in scalable video and generates more detailed metric for a given section of video.

\section{Browsing Interface}

Having the user's experience at the centre of our browsing interface design task, our main aim is to generate an intuitive and easily-readable video summary by conveying the significance of a shot from analysed videos via the size of its key-frame representation. In our case, the objective is to clearly present visual content that is dominant throughout the analysed section of the video, as well as to highlight some unanticipated content.

\subsection{Estimating key-frame significance}

Following the approach described above, where the size of a key-frame represents its summarisation significance, a cost function $C(i)$ that represent the desired frame size in the final layout is generated, where $C(i), i=1, \ldots, N$, $C(i) \in[0,1]$ and $N$ is the number of extracted key-frames for a given sequence. In order to evaluate the cost function in a way that will support the users visual experience of the final layout, the clustering based on perceptual 


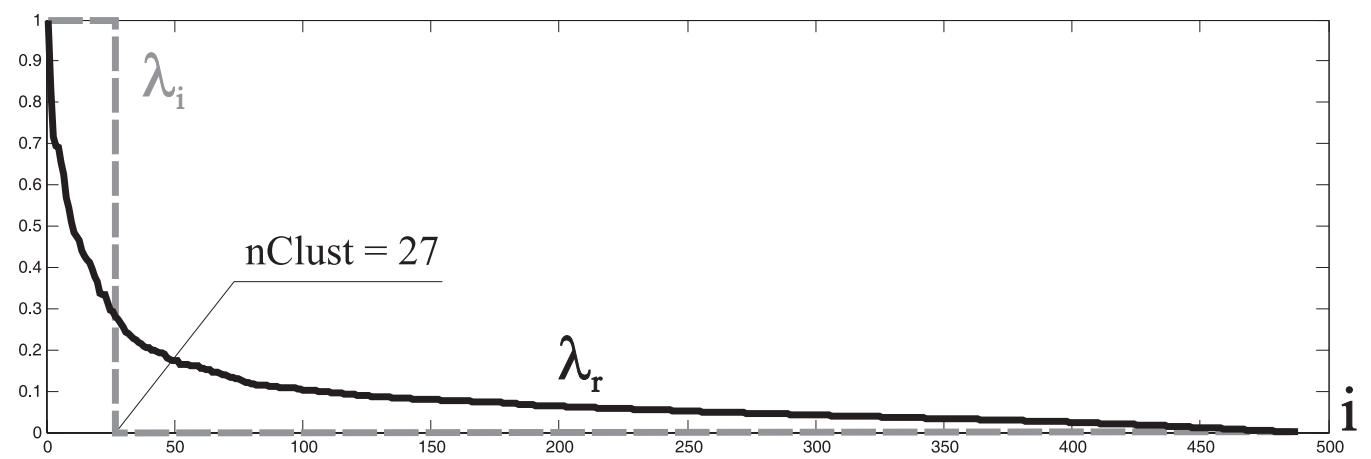

Fig. 3. Sorted eigenvalues of affinity matrix with estimated number of data clusters $n$ Clust in the ideal case $\left(\lambda_{i}\right)$ and a real case $\left(\lambda_{r}\right)$. By clustering eigenvalues in two groups, the number of points where $\lambda_{i}=1$ can be estimated.

similarity is used. More specifically, the self-tuning K-way spectral clustering approach [26] that utilises $18 \times 3 \times 3 \mathrm{HSV}$ colour histogram is applied. This approach enables unsupervised analysis of inherent structure of the key-frame data and it copes well with non-linearity of cluster shapes [39].

In order to estimate the the number of clusters an efficient and robust approach presented in [21] is used. It follows the idea that in an ideal case where the eigenvalues of the affinity matrix converge towards either 1 (high values) or 0 (low values), the size of high-value group gives a good estimate of number of clusters. In a real case, convergence to those extreme values will deteriorate, but there will be two opposite tendencies and thus two groups in the eigenvalue set, as depicted in Figure 3. Thus, the K-means clustering method is applied on sorted eigenvalues, where $K=2$ and initial locations of cluster centres are set to 1 for high-value cluster and 0 to low-value cluster. After clustering, the size of a high-value cluster gives a reliable estimate of number of clusters $k$ in analysed dataset.

Having grouped the key-frames into $k$ clusters, the maximum cost function $C(i)=h_{\max }$ is assigned to the key-frame closest to the centre of the corresponding cluster, thus representing the dominant content in the selected section of video. Other key-frames get assigned a cost as follows:

$$
C(i)=\beta \cdot\left(1-e^{-\frac{d(i)^{2}}{2 \sigma_{i}^{2}}}\right) \cdot h_{\max }
$$

where $d(i)$ is the $i^{\text {th }}$ frame's distance to the central frame and $\sigma_{i}$ is the variance of the cluster. The parameter $\beta$ controls the balance between the importance of the cluster centre and its outliers, and it is set empirically to 0.7. 


\subsection{Layout}

The main task of the layout module is to generate a visual summary that optimally follows the values of the cost function by using only frame sizes available in comic-like panel templates. Precision of this approximation depends upon the maximum height of a panel $h_{\max }$ which gives granularity of the solution. For a given $h_{\max }$, a set of panel templates is generated, assigning a vector of frame sizes to each template, as presented in more detail in [40].

Since the aim here is to optimally utilise the available space given the required sizes of images, this is a problem of discrete optimisation. However, unlike many well studied problems like stock cutting or bin packing [41], there is a non-linear transformation layer of panel templates between the error function and available resources. Therefore, a sub-optimal solution using dynamic programming [42] is proposed. In the Section 5 the experimental results of the proposed sub-optimal solution show that deviation from the optimal results can be practically disregarded.

The proposed method follows a typical structure of dynamic programming algorithm by efficiently finding the solution to an optimisation problem in case the variables in the evaluation function are not interrelated simultaneously [21]. Although there is an indirect dependency between non-adjacent panels due to the fact that the width of the last panel is directly dependent upon the sum of widths of previously used panels, by introducing specific corrections to the DP error function the sub-optimal solution often achieves optimal results. The correction occurs when the last panel is added to the solution, and thus the current summary width $W_{\text {curr }}$ falls into one of the following categories:

- if $W_{\text {curr }}>W$, penalise with maximal error value

- if $W_{\text {curr }}=W$, calculate standard error function

- if $W_{\text {curr }}<W$, error function is recalculated for the row resized to fit required width $W$.

\section{$5 \quad$ Results}

The results presented in this section follow the steps of the proposed framework for video analysis. In the conducted experiments the TRECVID 2006 evaluation content has been used. This content is provided by NIST as the benchmarking material for evaluation of video retrieval systems. The videos from provided set were transcoded to scalable video format in order to achieve flexible representation needed for fast adaptation. For proposed video analysis the scalable representation enables easy access to different scales of motion 

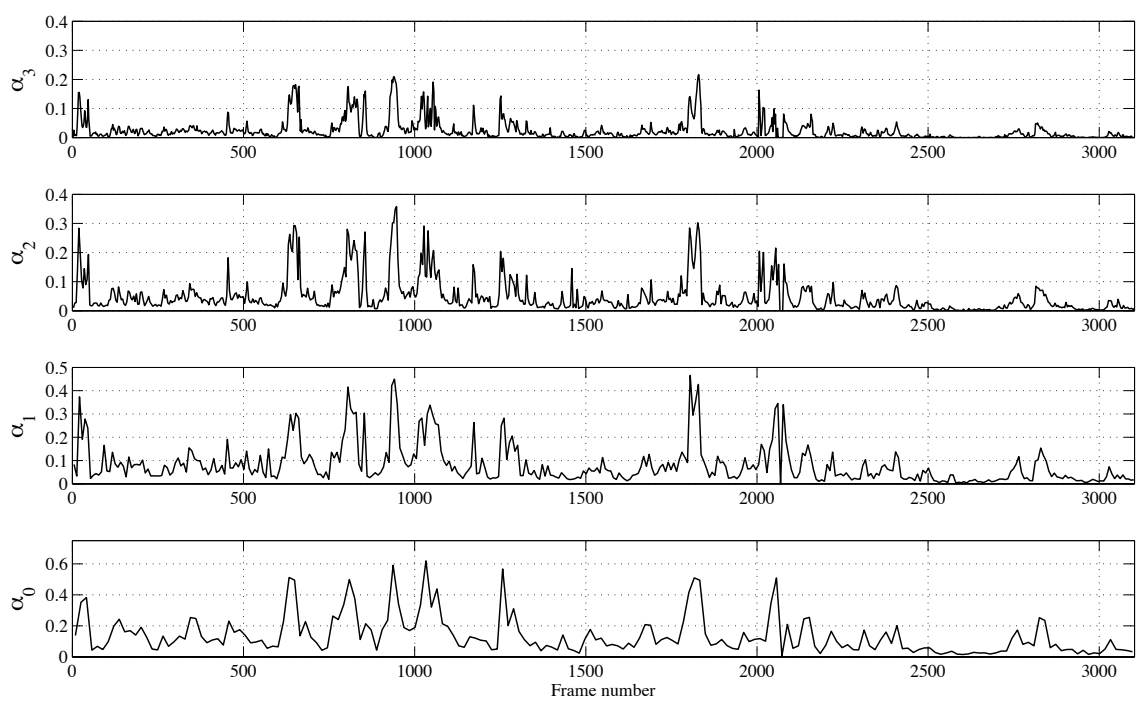

Fig. 4. Motion activity metric directly extracted at four different complexity levels of the first two minutes of the TRECVID test sequence MRS042546

information.

An example of the proposed motion activity metric $\alpha_{s}$, where $s$ represents the motion layer, extracted from scalable video is presented in Figure 4 . The video was encoded in 5 temporal layers, as in Figure 1. Therefore the motion information within compressed video is available at 4 different scales. Note that in the metric extraction step the full decoding of video is not needed since the position of motion information within the compressed video can be easily located. Therefore only entropy decoding of motion information from the selected layer has been performed and the motion activity measure has been computed. While the number of motion compensated frames in different layers depends on the level of temporal decomposition, the frame numbers in Figure 4 are related to the original sequence.

Decrease of motion activity amplitudes from lower to higher scales is present due to larger spatial shift of object at lower frame-rates. However, the results for motion activity over different scales show high correlation. Although the results from the lowest layer $(s=0)$ provide the coarsest sampling in motion activity space, it is already obvious which parts of video sequence already have highest motion activity. More precise information on the activity is available from higher layers. At higher levels it can often be seen that a part of the sequence with more constant activity is broken into parts with more diverse events. While higher levels provide more details, the complexity of decoding and activity evaluation grows by factor 2 from lower to higher levels.

The results depicted in the Figure 5 represent a scale space at four stages 

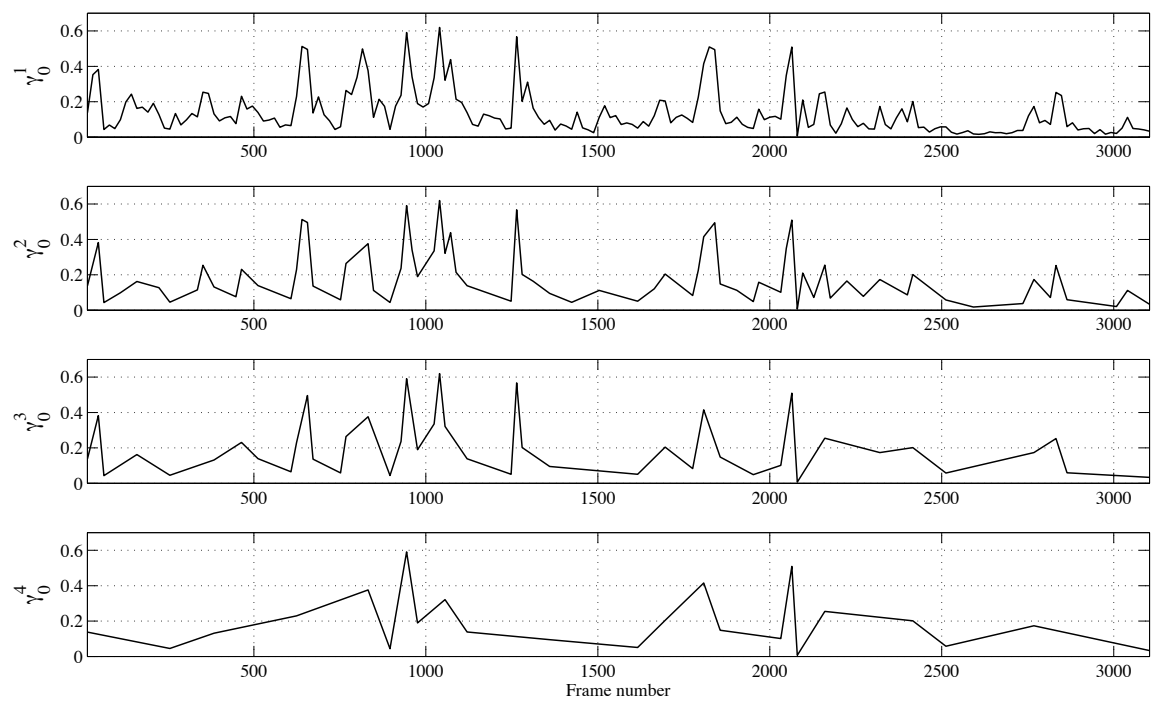

Fig. 5. Scale space of the temporal descriptor generated by the DCE algorithm for the sequence in Figure 4

of the DCE simplification process, applied to the metric $\alpha_{0}$ in Figure 4 . The constant location and value of the dominant features at all scales can be observed, as well as gradual removal of less important features of the metric curve. The bottom curve $\gamma_{0}^{4}$ is used in key-frame selection, and its final layout is presented in Figure 7.

In order to evaluate the results of the DP sub-optimal panelling algorithm, results are compared against the optimal solution, which searches for all possible combinations of summary layout and finds the optimal solution for given cost function. The search space is created by efficiently generating [43] all compositions of $W$ that fit $\mathcal{N}$ into the layout. Results in Table 1 show the dependency of approximation error $\Delta$ for two main algorithm parameters: maximum row height $h_{\max }$ and number of frames in the summary $\mathcal{N}$. The approximation error is calculated as:

$$
\Delta=\frac{1}{\mathcal{N} \cdot h_{\max }}\left(\sum_{i=1}^{\mathcal{N}}(C(i)-\Theta(i))^{2}\right)^{1 / 2}
$$

As expected, error generally drops as both $h_{\max }$ and $\mathcal{N}$ rise. For summary sizes used in this experiment, the optimal height of panel templates $h_{\max }$ depends on the number of frames $\mathcal{N}$. Due to boundary effects for small $\mathcal{N}$ and thus a small number of clusters involved, this result is different from results we measured for large summaries with $\mathcal{N}>100$ (see [21]). As we described in Section 4.2 , the reason behind this phenomenon is the finite layout width $W$, that results in sub-optimal solution of the DP algorithm. The approximation 
Table 1

\begin{tabular}{|c||c|c|c|c|c|c|}
\hline$h_{\max } \backslash \mathcal{N}$ & 7 & 10 & 15 & 20 & 30 & 40 \\
\hline \hline 1 & 8.57 & 7.92 & 6.42 & 5.66 & 5.89 & 5.34 \\
2 & 3.74 & 3.48 & 3.01 & 2.91 & 2.37 & 2.20 \\
3 & 4.09 & 3.88 & 3.12 & 2.39 & 2.30 & 2.03 \\
4 & 4.21 & 5.62 & 4.65 & 3.27 & 2.33 & 2.14 \\
\hline
\end{tabular}

Approximation error $\Delta$ as a function of maximum row height $h_{\max }$ and number of frames $\mathcal{N}$, expressed in $[\%]$

Table 2

\begin{tabular}{|c||c|c|c||c|c|c|}
\hline \multicolumn{1}{|c||}{} & \multicolumn{3}{c||}{$\Delta_{\text {optimal }}$} & \multicolumn{3}{c|}{$\Delta_{\text {DP }}-\boldsymbol{\Delta}_{\text {optimal }}$} \\
\hline$h_{\text {max }} \backslash \mathcal{N}$ & 7 & 10 & 15 & 7 & 10 & 15 \\
\hline \hline 1 & 8.57 & 7.92 & 6.42 & 0.00 & 0.00 & 0.00 \\
2 & 3.53 & 3.21 & 2.71 & 0.21 & 0.27 & 0.30 \\
3 & 3.78 & 3.61 & 2.99 & 0.31 & 0.27 & 0.13 \\
4 & 3.85 & 5.31 & 4.26 & 0.36 & 0.31 & 0.39 \\
\hline
\end{tabular}

Comparison of optimisation error for given layout parameters, expressed in [\%]

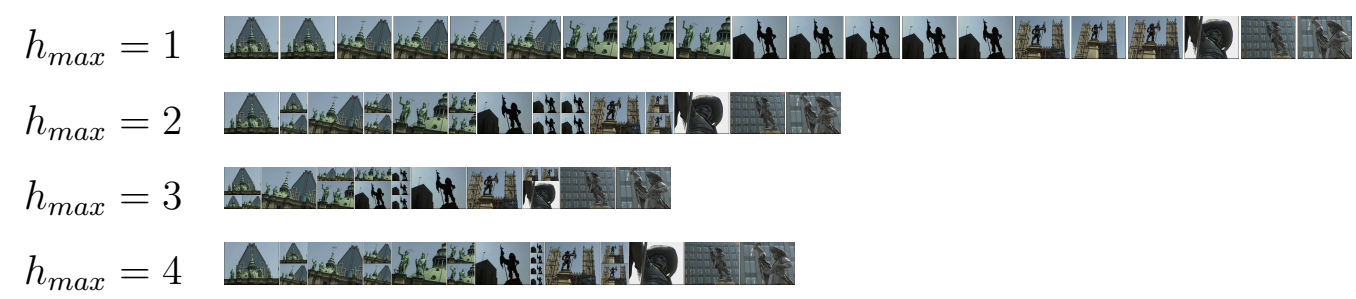

a) Resulting layouts using panels with different heights

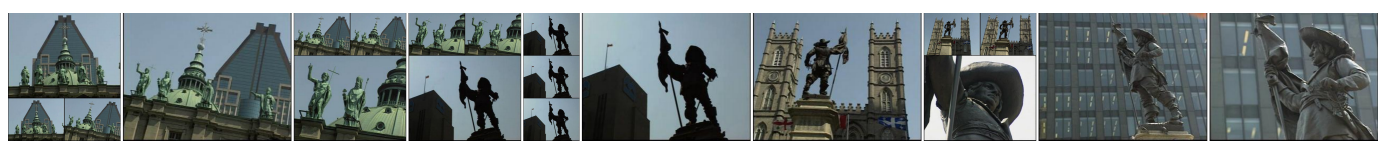

b) Enlarged layout for $h_{\max }=3$

Fig. 6. Resulting summaries for TRECVID test sequence FRANC101

error rises with $h_{\max }$ for lower values of $\mathcal{N}$, due to a strong boundary effect of our sub-optimal solution for small values of $W$, while for larger $\mathcal{N}$, larger panels produce better optimisation results. In addition, the spatial compression can vary for different $h_{\max }$, as depicted in Figure 6. Nevertheless, one can observe the spatial compression achieved by using proposed comic-like layout, by comparing it to the width of a typical summary, where $h_{\max }=1$ (top row of Figure 6). Without loosing the notion of temporal structure as well as representing every details of the content, the resulting video summary achieves spatial compression ratio of $2 / 5$ while producing visually pleasant experience for the user. 

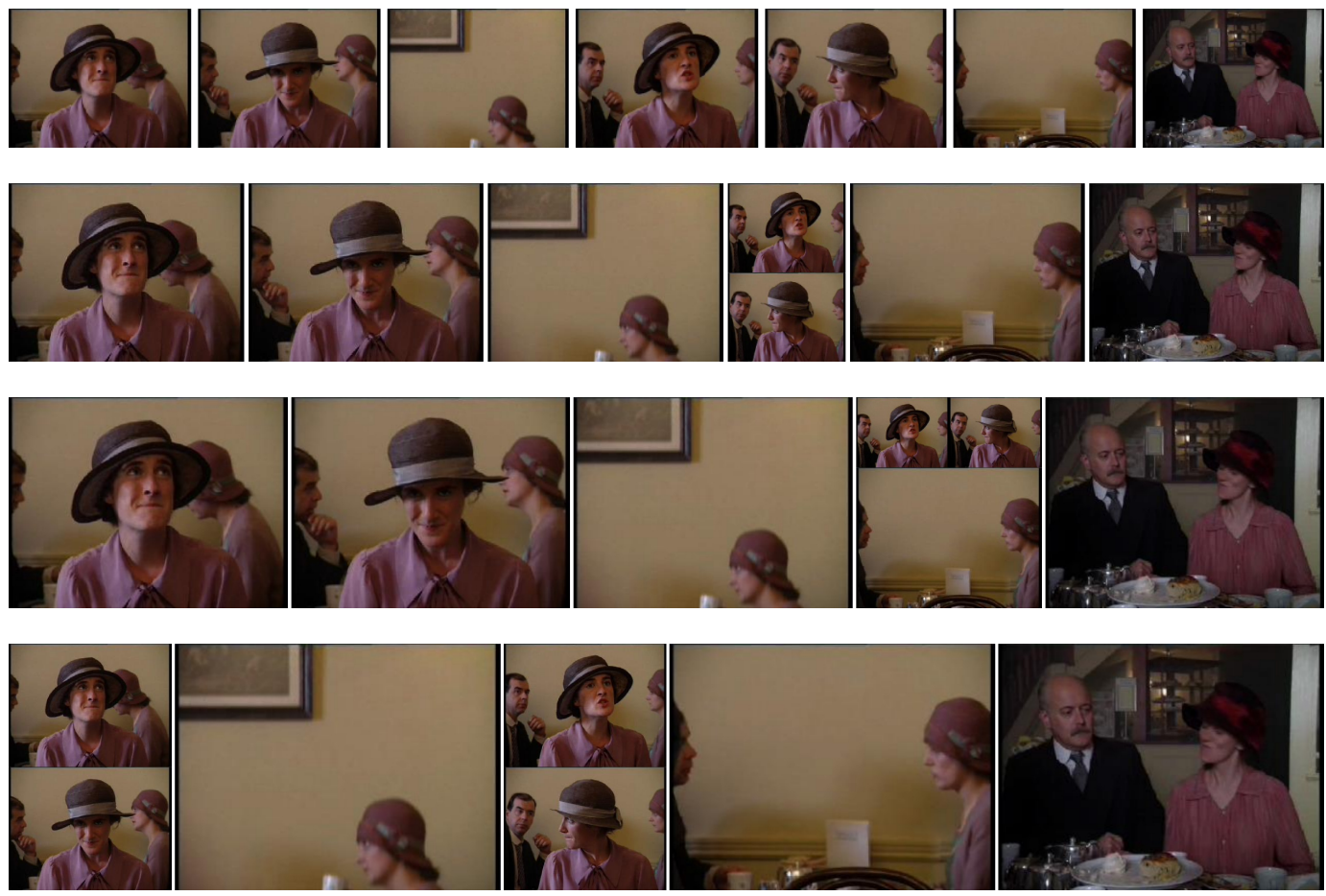

Fig. 7. Four video summaries of the TRECVID test sequence MRS042546 for $h_{\max }=1, \ldots, 4$

As presented in Figure 7, the main events of the analysed sequence: the two occurrences of the main actress leaving the scene as well as the shot change by the end of the sequence, have been captured and represented in the final summary that comprises only 7 frames. In addition, in the compact summaries, perceptually similar and temporarily adjacent frames have been less noticeable than the representatives of the more distinctive events.

In the Table 2, the first three columns show the approximation error of the optimal method, while the other three columns show absolute difference between errors of the optimal and sub-optimal solutions. The overall error due to the sub-optimal model is on average smaller than $0.3 \%$ of the value of cost function. Therefore, the error can be disregarded and this result shows that the much faster sub-optimal solution achieves practically the same results with the optimal method. The optimal algorithm lays out 40 frames on a page in approximately 2 minutes, while the sub-optimal algorithm does it in a fraction of a second.

Being a part of the system with the highest complexity ( $N P$ hard), the layout optimisation is evaluated in terms of processing speed. The approach presented in [44], as well as the optimal solution used in comparison of approximation error, regardless of the speedup achieved by various heuristics [24], have been proved not feasible for larger summaries. The layout times for of the algorithms presented in [44] $\left(T_{O R I G}\right)$ and [24] $\left(T_{F A S T}\right)$, compared to the proposed 


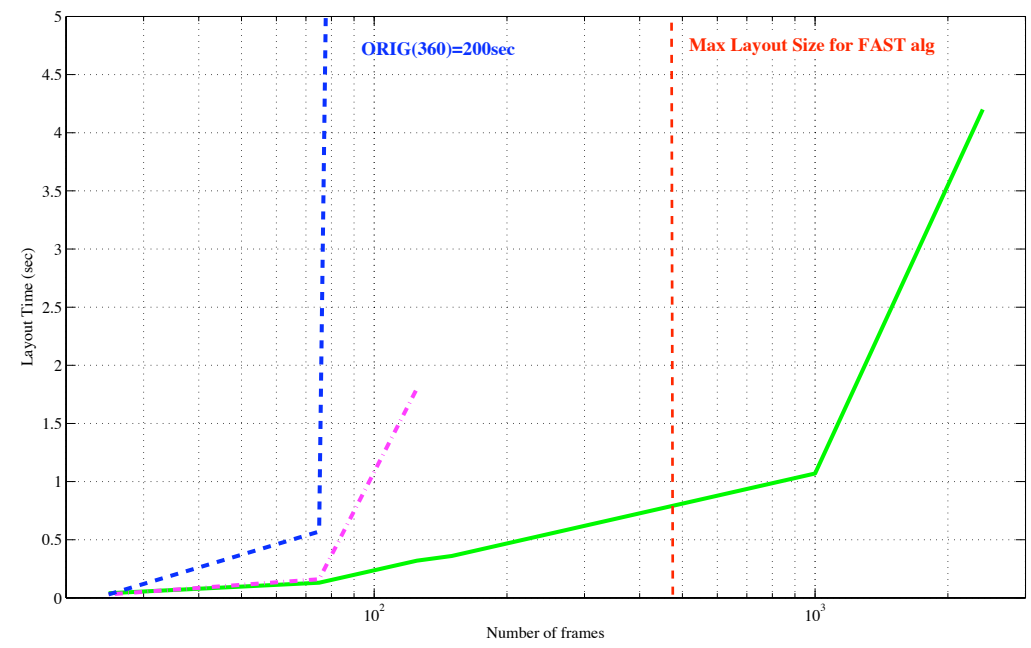

Fig. 8. Comparison of the layout speed for methods presented in [44] [ORIG], [24] [FAST] the proposed method [DPLY].

\begin{tabular}{|c||c|c|c|}
\hline $\mathcal{N}$ & $T_{\text {ORIG }}$ & $T_{F A S T}$ & $T_{D P L Y}$ \\
\hline \hline 25 & 0.03 & 0.03 & 0.04 \\
\hline 75 & 0.57 & 0.16 & 0.13 \\
\hline 125 & 200 & 1.8 & 0.32 \\
\hline 150 & $\times$ & $\times$ & 0.44 \\
\hline 1000 & $\times$ & $\times$ & 1.07 \\
\hline 2400 & $\times$ & $\times$ & 4.20 \\
\hline
\end{tabular}

Table 3

Comparison of layout algorithm speeds, depending upon number of frames $\mathcal{N}$.

method $\left(T_{D P L Y}\right)$ are depicted in Fig. 8 and numerically given in Table 3. From the results shown it can be observed that the utilised method achieves linear complexity and thus proves to be suitable for fast summarisation and adaptive browsing interfaces.

\section{Conclusions}

This paper presents an efficient summarisation and browsing framework for scalable video that utilises inherently hierarchical compressed domain motion features and creates summaries using a fast algorithm for temporal analysis. The system exploits the narrative structure of comics using well-known intuitive rules, creating visual summaries in an efficient and user centred way.

Proposed video analysis method is based on the hierarchical extraction of 
motion information from scalable videos. The motivation for such approach follows the observation that the motion information in scalable video coding is organised in hierarchical way for the purpose of scalability, providing easily accessible basis for fast analysis. The first stage of video analysis - evaluation of the motion activity for motion compensated frames has been realised novel metric based on the deviation of motion intensity. The activity metric links motion information used in video coding with the analysis stage. Therefore it has been carefully designed with a goal to bridge the gap between most of available motion compensation features and the analysis. The proposed activity metric uses weighting for variable size motion units providing support for flexible motion models used in efficient video compression. Moreover, it reuses compensation parameters of differently predicted motion units enabling activity evaluation using all inter coded modes.

Employing a fast and robust curve simplification algorithm, a scale space of temporal descriptors is generated. Using the descriptor scale space, the size of summary, i.e. number of frames in the final layout, can be customised. Given a spatial constraints, a fast layout algorithm generates a comic-like summary of the video sequence using a fast discrete optimisation method. Not only does this approach improves the processing time of the summarisation task, but it enables new functionalities of visualisation for large-scale video archives, such as runtime interaction, scalability, and relevance feedback. In addition, a set of high-level rules of comics grammar can be exploited to improve representation of time to further improve compactness of generated browsing interface.

\section{Acknowledgement}

The work presented was developed within VISNET II, a European Network of Excellence (http://www.visnet-noe.org), funded under the European Commission IST FP6 programme.

\section{References}

[1] N. Adami, A. Signoroni, R. Leonardi, State-of-the-art and trends in scalable video compression with wavelet-based approaches, Circuits and Systems for Video Technology, IEEE Transactions on 17 (9) (2007) 1238-1255, 1051-8215.

[2] H. Schwarz, D. Marpe, T. Wiegand, Overview of the scalable video coding extension of the H.264/AVC standard, Circuits and Systems for Video Technology, IEEE Transactions on 17 (9) (2007) 1103-1120, 1051-8215. 
[3] M. Mrak, E. Izquierdo, Scalable video coding, in: B. Furht (Ed.), Encyclopedia of Multimedia, 1st Edition, Springer, 2006.

[4] A. Divakaran, R. Radhakrishnan, K. A. Peker, Video summarization using descriptors of motion activity: a motion activity based approach to key-frame extraction from video shots, Journal of Electronic Imaging 10 (2001) 909-916.

[5] J. Bescós, J. M. Martínez, L. Herranz, F. Tiburzi, Content-driven adaptation of on-line video, Image Commun. 22 (7-8) (2007) 651-668.

[6] S. Xinding, B. S. Manjunath, A. Divakaran, Representation of motion activity in hierarchical levels for video indexing and filtering, in: Image Processing. 2002. Proceedings. 2002 International Conference on, Vol. 1, 2002, pp. I-149-I-152 vol.1.

[7] A. Divakaran, R. Radhakrishnan, K. A. Peker, Motion activity-based extraction of key-frames from video shots, in: Image Processing. 2002. Proceedings. 2002 International Conference on, Vol. 1, 2002, pp. I-932-I-935 vol.1.

[8] W. Wolf, Key frame selection by motion analysis, in: Acoustics, Speech, and Signal Processing, 1996. ICASSP-96. Conference Proceedings., 1996 IEEE International Conference on, Vol. 2, 1996, pp. 1228-1231 vol. 2.

[9] V. Kobla, D. S. Doermann, K.-I. Lin, C. Faloutsos, Compressed-domain video indexing techniques using DCT and motion vector information in MPEG video, in: I. K. Sethi, R. C. Jain (Eds.), Proc. SPIE Vol. 3022, p. 200-211, Storage and Retrieval for Image and Video Databases V, Ishwar K. Sethi; Ramesh C. Jain; Eds., Vol. 3022 of Presented at the Society of Photo-Optical Instrumentation Engineers (SPIE) Conference, 1997, pp. 200-211.

[10] L. Jae-Ho, L. Gwang-Gook, K. Whoi-Yul, Automatic video summarizing tool using MPEG-7 descriptors for personal video recorder, Consumer Electronics, IEEE Transactions on 49 (3) (2003) 742-749, 0098-3063.

[11] A. Hanjalic, Generic approach to highlights extraction from a sport video, in: IEEE International Conference on Image Processing (ICIP'03), Vol. 1, Barcelona, Spain, 2003, pp. 1-4.

[12] N. Chong-Wah, M. Yu-Fei, Z. Hong-Jiang, Automatic video summarization by graph modeling, in: Computer Vision, 2003. Proceedings. Ninth IEEE International Conference on, 2003, pp. 104-109 vol.1.

[13] N. Chong-Wah, M. Yu-Fei, Z. Hong-Jiang, Video summarization and scene detection by graph modeling, Circuits and Systems for Video Technology, IEEE Transactions on 15 (2) (2005) 296-305.

[14] D. Gibson, N. Campbell, B. Thomas, Visual abstraction of wildlife footage using gaussian mixture models and the minimum description length criterion, in: R. Kasturi, D. Laurendeau, C. Suen (Eds.), International Conference on Pattern Recognition, IEEE Computer Society, 2002, pp. 814-817. 
[15] D. DeMenthon, V. Kobla, D. Doermann, Video summarization by curve simplification, in: MULTIMEDIA '98: Proceedings of the sixth ACM international conference on Multimedia, ACM Press, New York, 1998, pp. 211218.

[16] L. J. Latecki, R. Lakamper, Convexity rule for shape decomposition based on discrete contour evolution, Computer Vision and Image Understanding 73 (1999) 441-454(14).

[17] J. Calic, E. Izquierdo, A multiresolution technique for video indexing and retrieval, in: Image Processing. 2002. Proceedings. 2002 International Conference on, Vol. 1, 2002, pp. I-952-I-955 vol.1.

[18] T. Liu, X. Zhang, J. Feng, K.-T. Lo, Shot reconstruction degree: a novel criterion for key frame selection, Pattern Recognition Letters 25 (12) (2004) 1451-1457.

[19] Z. Yueting, R. Yong, T. S. Huang, S. Mehrotra, Adaptive key frame extraction using unsupervised clustering, in: Image Processing, 1998. ICIP 98. Proceedings. 1998 International Conference, Vol. 1, 1998, pp. 866-870.

[20] A. Hanjalic, Z. HongJiang, An integrated scheme for automated video abstraction based on unsupervised cluster-validity analysis, Circuits and Systems for Video Technology, IEEE Transactions on 9 (8) (1999) 1280-1289.

[21] J. Calic, D. P. Gibson, N. W. Campbell, Efficient layout of comic-like video summaries, IEEE Transactions on Circuits and Systems for Video Technology 17 (7) (2007) 931-936.

[22] R. Dony, J. Mateer, J. Robinson, Techniques for automated reverse storyboarding, Vision, Image and Signal Processing, IEE Proceedings- 152 (4) (2005) 425-436.

[23] S. Uchihashi, J. Foote, A. Girgensohn, J. Boreczky, Video manga: generating semantically meaningful video summaries, in: MULTIMEDIA '99: Proceedings of the seventh ACM international conference on Multimedia, ACM Press, New York, NY, USA, 1999, pp. 383-392.

[24] A. Girgensohn, A fast layout algorithm for visual video summaries, in: Multimedia and Expo, 2003. ICME '03. Proceedings. 2003 International Conference on, Vol. 2, 2003, pp. 77-80.

[25] A. Y. Ng, M. I. Jordan, Y. Weiss, On spectral clustering: Analysis and an algorithm (2002) 849-856.

[26] L. Zelnik-Manor, P. Perona, Self-tuning spectral clustering (2005) 1601-1608.

[27] JPEG 2000 image coding system, ISO/IEC 15444.

[28] D. S. Taubman, M. W. Marcellin, JPEG 2000: Image Compression Fundamentals, Standards and Practice, Kluwer Academic Publishers, Norwell, MA, USA, 2001. 
[29] S.-T. Hsiang, J. W. Woods, Embedded video coding using invertible motion compensated 3-d subband/wavelet filter bank, Signal Processing: Image Communication 16 (8) (2001) 705-724.

[30] A. Secker, D. Taubman, Lifting-based invertible motion adaptive transform (LIMAT) framework for highly scalable video compression, Image Processing, IEEE Transactions on 12 (12) (2003) 1530-1542, 1057-7149.

[31] Y. Andreopoulos, A. Munteanu, J. Barbarien, M. Van der Schaar, J. Cornelis, P. Schelkens, In-band motion compensated temporal filtering, Signal Processing: Image Communication 19 (7) (2004) 653-673.

[32] N. Sprljan, M. Mrak, G. Abhayaratne, E. Izquierdo, A scalable coding framework for efficient video adaptation, in: 6th International Workshop on Image Analysis for Multimedia Interactive Services, WIAMIS 2005, Vol. 1, 2005.

[33] Coding of audiovisual objects - part 10: Advanced video coding, ISO/IEC 14 496-10.

[34] MPEG press release, ISO/IEC JTC 1/SC 29/WG 11 N9159, 2007.

[35] H. Schwarz, D. Marpe, T. Wiegand, Analysis of hierarchical B pictures and MCTF, in: Multimedia and Expo, 2006 IEEE International Conference on, 2006, pp. 1929-1932.

[36] M. Mrak, N. Sprljan, E. Izquierdo, Motion estimation in temporal subbands for quality scalable motion coding, Electronics Letters 41 (19) (2005) 1050-1051.

[37] M. Wien, Variable block-size transforms for H.264/AVC, Circuits and Systems for Video Technology, IEEE Transactions on 13 (7) (2003) 604-613, 1051-8215.

[38] T. Wiegand, H. Schwarz, A. Joch, F. Kossentini, G. J. Sullivan, Rateconstrained coder control and comparison of video coding standards, Circuits and Systems for Video Technology, IEEE Transactions on 13 (7) (2003) 688$703,1051-8215$.

[39] A. K. Jain, M. N. Murty, P. J. Flynn, Data clustering: a review, ACM Computing Surveys 31 (3) (1999) 264-323.

[40] J. Calic, N. Campbell, Compact visualisation of video summaries, EURASIP Journal on Advances in Signal Processing 2007 (2007) Article ID 19496, 14 pages.

[41] A. Lodi, S. Martello, M. Monaci, Two-dimensional packing problems: A survey, European Journal of Operational Research 141 (2) (2002) 241-252.

[42] R. E. Bellman, S. E. Dreyfus, Applied Dynamic Programming, Princeton University Press, Princeton, NJ, 1962.

[43] A. Nijenhuis, H. S. Wilf, Combinatorial algorithms: for computers and calculators, 2nd Edition, Computer science and applied mathematics, Academic Press, New York; London, 1978. 
[44] S. Uchihashi, J. Foote, Summarizing video using a shot importance measure and a frame-packing algorithm, in: Acoustics, Speech, and Signal Processing, 1999. ICASSP '99. Proceedings., 1999 IEEE International Conference on, Vol. 6, 1999, pp. 3041-3044. 